\title{
Panoramic radiography in dentistry
}

\author{
Ingrid Różyło-Kalinowska ${ }^{1}$ D
}

Received: 9 August 2021 / Accepted: 5 October 2021 / Published online: 7 December 2021

(c) The Author(s) 2021

\begin{abstract}
Panoramic radiography is an extraoral radiography modality that provides twodimensional information about the teeth and the maxillofacial skeleton. It is a valuable adjunct for diagnosis and treatment planning as it facilitates one-time imaging of all teeth, the mandible, parts of maxilla including a large part of the maxillary sinus, hard palate and temporomandibular joints (TMJs). As a tomographic image is prone to errors and artefacts, a good quality radiograph in most patients can be achieved by following the standard rules and through proper patient positioning. In this article, we will discuss indications for panoramic radiography imaging, steps in taking the radiograph, as well as limitations, pitfalls and complications of the procedure. Tomographic imaging of temporomandibular joint is also discussed.
\end{abstract}

Keywords Panoramic radiography · Tomography · Extraoral · TMJ tomography

\section{Quick reference/description}

Panoramic radiography is an extraoral radiography technique that provides twodimensional information about the teeth and the maxillofacial skeleton. It is a valuable adjunct for diagnosis and treatment planning as it facilitates one-time imaging of all teeth, the mandible, parts of maxilla including a large part of the maxillary sinus, hard palate and temporomandibular joints (TMJs). As a tomographic image is prone to errors and artefacts, a good quality radiograph in most patients can be achieved by following the standard rules and through proper patient positioning.

\section{Indications}

Indications for panoramic radiography imaging are:

Ingrid Różyło-Kalinowska

rozylo.kalinowska@umlub.pl

1 Department of Dental and Maxillofacial Radiodiagnostics, Medical University of Lublin, ul. Doktora Witolda Chodzki 6, 20-093 Lublin, Poland 
- Periodontal disease with simultaneous evaluation of all teeth and the extent of periodontal bone defects

- Orthodontic evaluation including presence of teeth germs, developmental stage of the dentition and the presence of retained, supernumerary or impacted teeth

- Impacted third molars

- Dental age estimation

- Lesions such as tumors, cysts, and other bone diseases that cannot be entirely imaged by periapical radiographs

- Mandibular trauma

- Implant planning

\section{Materials/instruments}

- Lead apron

- Bite piece

- Chin support

- Cotton roll (optional)

- Single use protective sleeve

- Disinfectant solution spray

- Step stool

- Panoramic machine

- Camera (optional)

- Ruler (optional for TMJ tomography)

\section{Procedure}

Panoramic radiography is the most prevalent extraoral radiography technique. It is a two-dimensional interpretation of tomographic images of curved structures. Panoramic radiography is indicated for the diagnosis of various physiological and pathological conditions of the oral and maxillofacial regions. It facilitates the one-time imaging of teeth, mandibular bone, parts of maxilla including a large portion of maxillary sinus, hard palate and both TMJs.

The referral for a panoramic radiograph should be justified by the indications and the radiation-risk evaluation. It should not be taken as a routine radiograph. As it is a tomographic radiograph, only structures that lie within the focal plane or trough are clearly visible, while other structures outside the focal plane are blurred or invisible (Fig. 1).

\section{Taking a panoramic radiograph}

Synchronized movement of the X-ray tube and the image detector in opposite directions results in an appropriate panoramic radiograph. Due to the application of a robotic $\mathrm{C}$-arm in a panoramic machine, the tomographic movement is 
Fig. 1 Diagram of focal trough

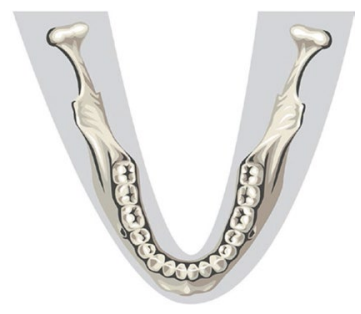

performed in a horizontal plane along a curve adjusted to the shape of the dental arches (Fig. 2). Its complex projection geometry along with rotational movement results in different shadows in the panoramic radiograph.

- Structures located within the focal trough have single shadows

- Single structures located adjacent to the midline and outside the focal trough have double real shadows

- Double structures closer to the radiographic edges outside the focal plane have ghost shadows

Advances in panoramic radiography depend on:

- Exposure time reduction

- Exposure dose reduction

- Increase in image sharpness

- Registration of several parallel thinner layers within the focal trough

- Automatic image post-processing for selecting best-quality segments within the layers to compile into a high-quality radiograph. An operator can also scroll through the layers and choose images as required.

Obtaining a panoramic radiograph is comparatively simple with proper patient positioning. If the appropriate steps are carefully followed, a good quality radiograph can be obtained in most patients.

Fig. 2 Diagram of rotational tomographic movement of X-ray tube and image sensor

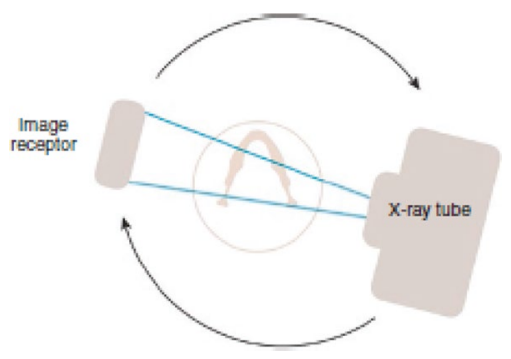




\section{Steps in obtaining a panoramic radiograph}

\section{Introductory Steps}

- Read and evaluate the referral prescription to ensure justification of the radiograph.

- Inform the patient and/or guardian about the radiation risk, and ensure that an informed consent is signed per applicable regulation.

- In female patients of child-bearing age, enquire about the possibility of pregnancy. Pregnancy is not an absolute contraindication for radiography but should be delayed until after birth, whenever possible.

- Generate or open a patient file in a dedicated software. The dental arch shape and exposure settings can be selected based on the software (Fig. 3). Entering the patient's gender and birth date leads to default settings that can be adjusted as required. A section of a panoramic radiograph should be selected instead of the whole radiograph if possible.

\section{Patient preparation}

- Instruct the patient to remove all metallic objects including removable orthodontic appliances, removable prostheses, piercings, glasses, necklaces, hearing aids and hair pins to prevent artefacts from the radiographed site.

- When the local legislation mandates the use of a lead apron during X-ray exposure, a lead apron with the longer side on the patient's back or a two-sided apron is used. If the patient's guardian accompanies the patient during the exposure, he/ she should also wear a lead apron.

\section{Patient positioning}

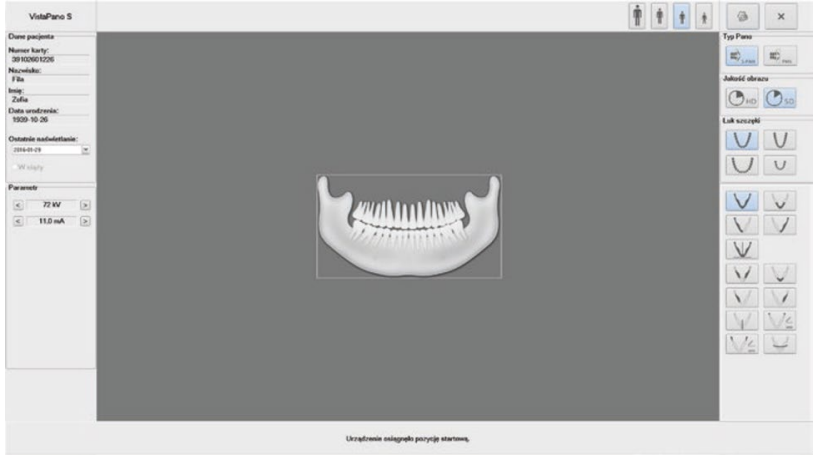

Fig. 3 Screenshot of software with choice of exposure parameters and shape of dental arches 
- A bite piece is selected according to the patient's dentition (Fig. 4a, b).

- In case the patient's teeth (anterior or more) are missing or mobile (due to periodontal bone disease) or the patient has sustained a trauma, the use of a chin support is advised (Fig. 4c, d).

- Use a sterile bite piece or cover the bite piece with a single use protective sleeve. Disinfect the chin support with a disinfectant solution spray.

- The level of the C-arm is adjusted to the patient's height using a positioning device.

- Instruct the patient to enter the $\mathrm{C}$-arm. Most machines have the ability to select a $\mathrm{C}$-arm position that makes it more convenient for the patient to enter it with minimal risk of colliding with the device elements. If the device has a dedicated seat, ask the patient to sit down. If the machine accommodates standing patients only, technique modification is required. Tall patients will need to sit on a stool with no back rest. A step stool is used for a small child. Patients in a wheelchair can be examined in machines without a seat, only if the wheels do not collide with the machine base. After stabilizing the patient in the machine, adjust the C-arm to the level of the patient's head.

- Instruct the patient to hold the handles (Fig. 4e). In standing patients, the patient may have to be asked to take half a step forward to straighten the cervical spine (Fig. 4f).

- Ask the patient to rest the chin on a regular chin support or on a special chin support for edentulous patients. The level of chin support should ensure that the patient's neck remains straight.

- A dentulous patient is instructed to bite on the sterile bite piece by articulating the incisal edges of the upper and lower incisors in the grooves on the bite piece.

- Patient positioning can be guided by laser lights (Fig. 4g).

- Symmetrical positioning of the head depends on the first laser light, which should lie between the upper central incisors. It is essential to keep in mind that facial soft tissues can be asymmetrical, and the dental arch midline may not correlate with midline of the patient's facial structures. In patients with missing

(a)

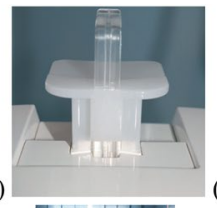

(b)

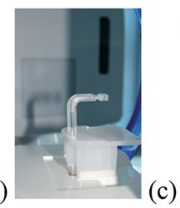

(f)

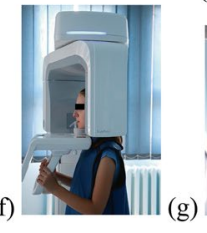

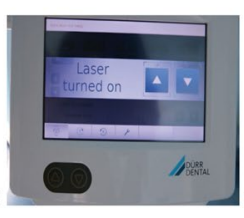

(h)

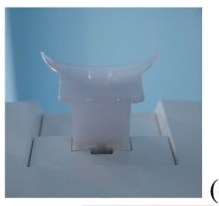

(d)

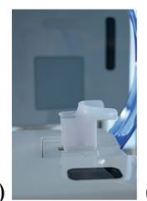

(e)
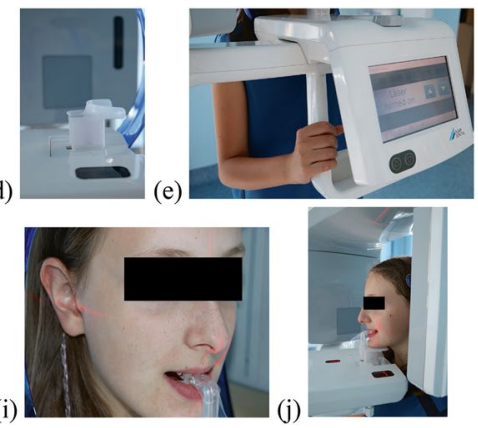

Fig. 4 Patient Positioning a, b Bite piece for dentate patients. c, $\mathbf{d}$ Chin support for edentulous patients. e Handles. f Adopting straight position. $\mathbf{g}$ Use of laser lights for positioning. $\mathbf{h}$ Symmetrical positioning of head using the first laser light. i Adjustment of Frankfort plane using the second laser light. j Selection of focal trough using the third laser light. 
anteriors or dental anomalies associated with the anterior teeth, positioning the first laser light can be difficult (Fig. 4h).

- A second laser light highlights the Frankfort plane. Proper positioning of the Frankfort plane ensures the horizontal alignment of the occlusal plane, which appears as a flat or a wide, flat 'U' (Fig. 4i).

- Cameras are fitted in some panoramic devices to facilitate positioning. In a camera image, the Frankfort plane is highlighted in color. For e.g., green for acceptable and red for incorrect positioning.

- Some panoramic machines also have a third laser light that helps to select the proper focal trough. It should extend between the upper lateral incisor and canine, and should fall on the angle of the mouth in edentulous patients (Fig. 4j). Verify the line of light in a lateral camera image or while the operator stands at the patient's side.

- Verify the correct position of the patient.

- After verifying the position, close the temporal supports by pressing a specific button or by pressing the 'Ready' button on the control panel.

\section{Patient instructions}

- Obtaining a high-quality radiograph requires proper fulfillment of instructions by the patient.

- Immediately prior to the exposure, instruct the patient the following (Table 1):

\section{X-ray exposure}

- Press the 'Ready' button on the control panel.

- Per local guidelines, the operator has to exit the X-ray room or protect herself/ himself behind a lead screen or a wall. He/she can also step away from the X-ray source to move away from the exposure area.

Table 1 Required patient actions before x-ray exposure

\begin{tabular}{ll}
\hline Actions & Rationale \\
\hline Swallow & $\begin{array}{l}\text { For prevention of swallowing during the exposure that may } \\
\text { cause motion artefacts } \\
\text { Close mouth }\end{array}$ \\
$\begin{array}{l}\text { For air removal from between open lips and to avoid radiolu- } \\
\text { cent shadow cast on images of incisors }\end{array}$ \\
Press the tongue against the hard palate & $\begin{array}{l}\text { To push the air inside from the mouth to prevent flat crescent- } \\
\text { shaped radiolucent shadows that overlap the periapical } \\
\text { regions of maxillary teeth and interfere with diagnosis of } \\
\text { periapical lesions }\end{array}$ \\
Breathe normally during exposure & $\begin{array}{l}\text { Few patients cannot hold their breath for a long period } \\
\text { No movement during exposure }\end{array}$ \\
\hline
\end{tabular}


- Press the exposure button and hold it down during the exposure. There is a slight delay in X-ray production after pressing the exposure button. The exposure does not occur if it is just pressed and released. X-ray generation can be detected through audio or visual signals. For e.g., yellow light on the machine and the exposure button.

- During the exposure, observe the patient through a video camera screen or a lead window.

- Patient movement during X-ray exposure will mostly require a retake of the radiograph. Therefore, discontinue the X-ray generation. Certain devices provide a live image preview during exposure to cross-check for motion artefacts in the radiograph due to patient movement. Some panoramic devices can also apply patient movement compensation in a limited range of up to $1.5 \mathrm{~cm}$ and does not help in case of severe, sharp movements.

\section{After exposure}

- Allow the patient to exit the patient positioning area safely.

- Whenever applicable, remove their protective lead apron.

- Save the radiograph in the database.

- Prepare the panoramic device for the next patient by changing the single-use protective sleeve over the bite piece and disinfecting the parts of the machine touched by the patient using a disinfectant spray.

\section{Limitations and difficulties in panoramic radiography}

- In some patients, it is challenging to take a good diagnostic radiograph because of anatomic or pathologic variations or uncooperativeness in spite of technological advances like positioning devices and image registration.

- Artefacts and errors can occur at any stage of obtaining a panoramic radiograph.

- Panoramic radiographs cannot be obtained in unconscious patients, neonates and patients who cannot maintain a straight cervical spinal position even while sitting.

- Obtaining a proper panoramic radiograph requires that the patient follows staff instructions and to remain motionless during the exposure. Panoramic radiography is not advised in young children due to lack of justified indications at their age. Few countries also have a strict age limit (5 years), below which panoramic radiography is contraindicated as per local legal provisions.

- Involuntary movements such as tremors, jerks, tics and myoclonic seizures can lead to poor quality radiographs or ending of the exposure. In patients with movement disorders like Parkinson's disease, panoramic radiographs must be taken in devices applying patient movement compensation.

- In patients with cognitive limitations or elderly patients with dementia, obtaining a panoramic radiograph can be difficult due to lack of comprehension of staff instructions and difficulty in standing or remaining still during the X-ray exposure. 
- In patients with severe Class II or Class III malocclusion, placement of upper and lower incisors in the grooves of the bite piece is almost impossible. Therefore, only one jaw will be seen clearly in the resultant radiograph depending on whether the upper or lower teeth were placed within the focal trough. In such patients, systems that permit one-time registration of multiple thin layers within the focal trough are preferred.

- Severe facial skeletal asymmetry results in difficulty in positioning the left and right side of the face within the focal trough leading to only one side being seen clearly on the radiograph.

- Patients with advanced periodontal bone disease and tooth mobility may be unable to bite in the groove of the bite piece as mobile teeth tend to tilt while closing the mouth. In such scenarios, it is recommended to use a chin support with a cotton roll to separate the upper and lower incisors.

- Patients with lockjaw, swelling or injuries of tongue or lips following trauma may require the use of a chin support with a cotton roll.

\section{Tomographic radiographs of the TMJs}

The majority of panoramic machines also allow the recording of tomographic images of the TMJ in open and closed mouth positions. Machine and patient preparation are the same as for a panoramic radiograph. The TMJ imaging mode should be selected on the control panel or the screen. In case the patient has a prior axial skull radiograph, the angulation of the long axis of the condylar heads in relation to the coronal plane can be measured from the radiograph. These values should be entered into the software or control panel. Default values $\left(17^{\circ}\right)$ are applied when the angulation is unknown. A dedicated positioning device that provides stable support for the nose and also allows adequate mouth opening is used (Fig. 5a, b).

\section{Steps for taking a tomographic radiograph of the TMJs}

- The radiograph has two stages, first with the mouth closed, and the second with the mouth open. This sequence can be the opposite in other machines.

- In the first part, the mouth is closed with the teeth in complete intercuspation (Fig. 6a).

- The head is symmetrically positioned in the sagittal plane using the first laser light.

- Caudal tilting of the Frankfort plane to $5^{\circ}$ is performed (second laser light).

Fig. 5 a, b Nasal support for TMJ tomographic imaging

(a)

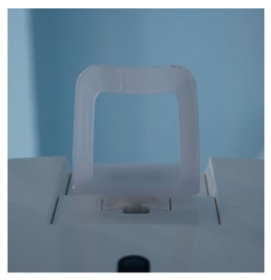

(b)

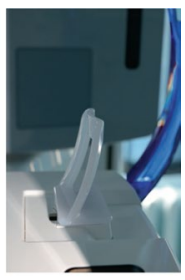


Fig. 6 Tomographic Radiograph of the TMJs. a Closed mouth position. b Open mouth position. (a)

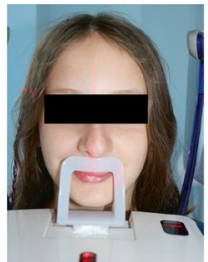

(b)

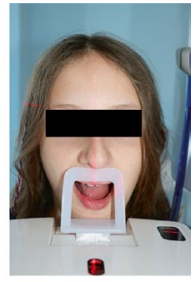

- The distance between the condylar head and the third laser light is measured with a ruler. This value is entered on the screen or control panel.

- Verify the head position, close the temporal supports and begin with the first stage of X-ray exposure.

- After the first exposure, instruct the patient to open her/his mouth wide without changing the head position (Fig. 6b).

- The value set for the distance should be reduced by $10 \mathrm{~mm}$ because the condylar heads shift out of the articular fossae in the open mouth position.

- Verify the head position and begin the second stage of exposure.

\section{Limitations of tomographic images of TMJs}

- With the advent of CBCT, TMJ tomographic imaging has now become obsolete.

- Quality of resultant radiograph is compromised by movement, lack of cooperation, inability to stand or sit still during exposure and inability to maintain a stable, straight neck position.

- Lockjaw

- The result is based on the TMJ anatomy as parasagittal slices are not always at right angles to the long axis of the condylar head particularly without a prior axial skull radiograph.

\section{Pitfalls \& complications}

- Retake of a panoramic radiograph leads to unjustified and additional exposure of the patient to ionizing radiation.

- Image magnification: apart from the standard records set by the manufacturer, image magnification varies even within the same radiograph, particularly due to positioning errors or anatomical issues. Image distortion in the horizontal plane is more advanced than in the vertical plane. Hence, although panoramic images are not indicated for linear measurements, they can be performed, if required, in the vertical plane.

- Tomographic character of image: the measurement of the focal trough is about $20 \mathrm{~mm}$ in lateral portions and about $10 \mathrm{~mm}$ in anterior region. As a panoramic radiograph clearly shows only those structures positioned within the focal trough, it is not adequately reliable to diagnose maxillary sinus diseases and midfacial fractures. Also, foreign bodies and pathological lesions located outside the focal 
trough are not visible (partially or completely) in the radiograph, which results in underdiagnosis.

- If the TMJ condyle axis does not align with the focal plane, it affects the condylar head image in a panoramic radiograph. A pseudocyst of the condyle is a prevalent artefact in panoramic radiography that mimics a cyst, which is actually a radiographic image of the concavity in the condylar process.

- Ghost and double shadows of normal anatomic structures complicate the anatomy of the panoramic radiograph.

\section{Further reading}

1. Rozylo-Kalinowska I. Imaging techniques in dental radiology, https://doi.org/10. 1007/978-3-030-41372-9_4 Panoramic radiography in dentistry

2. Langlais RP. Excercises in oral radiology and interpretation. 5th ed. St Louis: Saunders; 2016

3. Larheim TA, Westesson P-LA. Maxillofacial imaging. 2nd ed. Berlin: Springer; 2018

4. Whaites E, Drage N. Essentials of dental radiography and radiology. Edinburgh: Churchill Livingstone; 2013

5. White SC, Pharoah MJ. Oral radiology. principles and interpretation. St Louis: Mosby; 2014.

Open Access This article is licensed under a Creative Commons Attribution 4.0 International License, which permits use, sharing, adaptation, distribution and reproduction in any medium or format, as long as you give appropriate credit to the original author(s) and the source, provide a link to the Creative Commons licence, and indicate if changes were made. The images or other third party material in this article are included in the article's Creative Commons licence, unless indicated otherwise in a credit line to the material. If material is not included in the article's Creative Commons licence and your intended use is not permitted by statutory regulation or exceeds the permitted use, you will need to obtain permission directly from the copyright holder. To view a copy of this licence, visit http://creativecommons.org/ licenses/by/4.0/.

Publisher's Note Springer Nature remains neutral with regard to jurisdictional claims in published maps and institutional affiliations. 